\title{
New Dogs of War oder Peacekeeper von morgen? Argumente für und gegen die Beteiligung privater Militärfirmen an internationalen Friedensmissionen
}

\author{
Annina Bürgin*
}

\begin{abstract}
Scholars, NGOs, business, parliaments and governments are discussing whether private military companies (PMCs), which are numerous and offer quite diversified services, could contribute to peacekeeping missions, which face an immense lack of personnel. The article debates some of the triggers which have advanced the discussion and identifies several pros and cons regarding the contribution of PMCs to peacekeeping missions. In addition, discussions that took place in selected countries are outlined. It is conceivable that future debates will not revolve around whether peacekeeping tasks should be outsourced, but which duties, with whom and what monitoring and control tools should be applied.
\end{abstract}

Keywords: Private Militärfirmen, multilaterale Friedensmissionen, Vereinte Nationen

\section{Einleitung}

Das seit 2003 andauernde militärische Engagement der USA im Irak hat nicht nur die Tätigkeiten privater Sicherheitsfirmen (PSCs), sondern auch diejenigen von sogenannten privaten Militärfirmen (PMCs) ${ }^{1}$ ins öffentliche Licht gerückt. Schätzungen zufolge werden zwischen 25.000 und 70.000 Mitarbeiter der Sicherheitsindustrie im Zweistromland beschäftigt, wobei die amerikanische Regierung einer der größten Abnehmer entsprechender Dienstleistungen ist. Neben westlichen Regierungen beschäftigen aber auch zahlreiche Länder des Südens, multinationale Unternehmen, internationale Organisationen und Nichtregierungsorganisationen (NGOs) diese Firmen. Diese üben derart unterschiedliche Tätigkeiten aus, von der Bewachung von Ölfeldern oder Botschaften über diverse Logistikaufgaben für reguläre Armeen bis zur Betreibung von Kriegsgefangenenlagern, dass eine Zuordnung zu einer der gängigsten Kategorien schwierig ist. ${ }^{2}$ Könnte das breite und große Angebot der privaten Sicherheitsund Militärfirmen nicht auch für Aufgaben im Rahmen internationaler, vom UN-Sicherheitsrat abgesegneter PeacekeepingOperationen $(\mathrm{PKOs})^{3}$ genutzt werden? Diese Frage rückt zwei Entwicklungen der letzten Jahre ins Zentrum und verbindet sie. Erstens sind Friedensmissionen mit großen Herausforderungen konfrontiert, wobei vor allem die personellen Probleme noch zunehmen werden, da einerseits die Anzahl der PKOs - und damit der geforderten Truppen - stetig ansteigt und andererseits viele Staaten nach wie vor nicht bereit sind, mehr Soldaten zur Verfügung zu stellen als bisher. Zweitens hat sich mit den privaten Militär- und Sicherheitsfirmen in den 1990er Jahren ein neuer Wirtschaftssektor etabliert, des-

\footnotetext{
* Annina Bürgin, M.A., ist Doktorandin am Europainstitut der Universität Basel.

1 Die Begriffe PSCs oder PMCs werden nicht einheitlich gehandhabt; die Autorin verwendet die Bezeichnung private Militärfirma und meint damit Folgendes: Eine PMC ist eine privatrechtliche Unternehmung, die unterschiedlichsten Auftraggebern militärische, »combat-related « Dienstleistungen in High-Risk-Gebieten anbietet, wobei es auch zu sogenannten menschenrechtsintensiven Eingriffen kommen kann. Zur Entstehung und Entwicklung der PSMCs siehe u.a. Singer 2003, Avant 2005 oder Wulf 2005.

2 Eine oft verwendete Kategorisierung ist diejenige von Singer (2003: 88-100): Military Support Firms, Military Consultant Firms, Military Provider Firms.

3 Der hier verwendete Begriff meint »robustes« Peacekeeping im Sinne des Brahimi-Reports der UNO (United Nations 2000: 10).
}

sen Boom ungebremst anhält. Vertreter privater Militärfirmen beanspruchen für sich, dass sie die personellen Probleme der PKOs - zumindest partiell - lösen könnten, indem sie diverse Aufgaben von Friedensmissionen übernehmen. Dass die UNO private Unternehmem (nicht PMCs) anstellt, ist nicht neu. Jedoch wurden in letzter Zeit Aufgaben des Peacekeeping an PMCs vergeben. 1998 haben beispielsweise die USA Waffeninspekteure der Firma DynCorp unter Vertrag genommen und diese als amerikanischen Beitrag der OSCE Kosovo Verification Mission zur Verfügung gestellt (Bures 2005: 537). Der an der Börse notierte Großkonzern DynCorp führte unter anderem Helikoptertransporte für die PKO-Truppen der INTERFET in Osttimor durch, die zudem von der Firma Defence Systems Limited (heute ArmorGroup) sowohl im logistischen als auch im geheimdienstlichen Bereich unterstützt wurden (Singer 2003: 82; Bures 2005: 538). Außerdem werden Polizeikräfte in bestimmten PKOs der UNO eingesetzt, die aus privaten Militärfirmen rekrutiert sind. Sowohl für die MINUSTAH in Haiti als auch für die UNMIL in Liberia arbeiten Polizeieinheiten, die von der zu Lockheed Martin gehörenden Gruppe Pacific Architects \& Engineers im Auftrag der USA angestellt sind und der UNO zur Verfügung gestellt werden (PAE 2007; PAE 2007a). Wieso sollte also nicht verstärkt auf die Dienstleistungen der privaten Sicherheitsindustrie zurückgegriffen werden? Der erste Abschnitt dieses Artikels widmet sich Gründen, welche die Diskussionen ausgelöst haben. Im zweiten und dritten Absatz werden jeweils Argumente für und gegen den Einsatz von privaten Militärfirmen erörtert. Im vierten und letzten Abschnitt wird auf die Diskussionen in ausgewählten Staaten eingegangen. Die Schlussbemerkungen nehmen eine Einschätzung der angeführten Argumentationen vor.

\section{Auslöser der Diskussion}

Die in der UNO-Charta verankerten völkerrechtlichen Grundsätze sowohl der souveränen Gleichheit aller Mitglieder als auch des Interventionsverbots werden durch Interventionen - jeglicher Art - von außen strapaziert, da sich damit externe Akteure in die sogenannten inneren Angelegenheiten eines Staates einmischen. Das Spannungsverhältnis zwischen die- 
sen beiden Prinzipien und der internationalen Verpflichtung, fundamentale Menschenrechte zu schützen, ist in den letzten fünfzehn Jahren stärker geworden, da multilaterale, militärische Interventionen immer öfter mit Verweis auf die Verletzung von Menschenrechten gerechtfertigt wurden. Doch wer bestimmt, ob diese Interventionen zulässig sind? Die Einsätze der UNO werden vom UN-Sicherheitsrat verabschiedet und sind somit völkerrechtlich legitim - aber nicht demokratisch kontrolliert. Der intergouvernementalen Zusammenarbeit innerhalb der UNO und der Entscheidungsmacht des Sicherheitsrats ist ein Demokratiedefizit eigen, da es kein internationales Parlament gibt, das eine demokratische Kontrolle ausübt. Wenn internationale Einsätze von Streitkräften bezüglich der demokratischen Kontrolle nicht unproblematisch sind - wie steht es dann um die Kontrolle und Verantwortung eines privaten Unternehmens, das sicherheitsrelevante Aufgaben tätigt? Durch die Einbeziehung privater Akteure würde sich das Demokratiedefizit, das bei völkerrechtlich abgesegneten multilateralen Interventionen bereits besteht, noch verschärfen.

Folgende drei Triebkräfte, die sich teilweise gegenseitig verstärken, haben der Diskussion um die »Privatisierung des Friedens « - trotz demokratietheoretischer Bedenken - Antrieb verliehen. $^{4}$

Erstens haben sich die Streitkräfte in der zweiten Hälfte des 20. Jahrhunderts markant verändert. Einerseits wandelte sich die wahrgenommene Bedrohung und folglich das Aufgabenspektrum deutlich. Im Gegensatz zu der Zeit vor 1989 sind die potenziellen Feinde gegenwärtig weniger andere Staaten, sondern vor allem subnationale und/oder nichtstaatliche Akteure wie zum Beispiel terroristische Gruppierungen. Mit den einhergehenden neuen Herausforderungen haben sich die Aufgabenfelder der Streitkräfte verändert. Die im militärischen US-Jargon MOOTW (military operations other than war) genannten Operationen haben die typische Kriegführung abgelöst. Zunehmend werden Soldaten dafür eingesetzt, Streitparteien auseinanderzuhalten, gegen den weltweiten Drogenhandel zu kämpfen, Flüchtlinge zurückzuführen, Nahrungsmittel und Medikamente zu verteilen oder Schutz für humanitäre Organisationen zu leisten. Andererseits haben sich die Armeen zu kleineren, professionalisierten Armeen entwickelt. Dies hat zum einen mit der veränderten Bedrohungslage zu tun - die Gefahr eines Weltkriegs zwischen den zwei großen Blöcken USA und UdSSR ist vorbei. Zum andern führten Normenänderungen dazu, dass die Streitkräfte nach dem Ende des Ost-West-Konflikts stärker um ihre Legitimität innerhalb der Bevölkerungen zu kämpfen hatten, da die offensichtlichen Bedrohungen weggefallen sind. Schließlich hat die fortschreitende Technologisierung zu einer veränderten Kriegführung geführt. Diese tief greifenden Veränderungen der internationalen Politik und der Einstellungen der Bevölkerungen ebenso wie die stärker werdende Technologisierung führten in vielen westlichen Staaten zu einer Reduktion der Armeebestände und der Verteidigungsbudgets.

Zweitens ist, wie bereits erwähnt, die Zahl der multilateralen Interventionen in den letzten fünfzehn Jahren stetig und stark

4 Siehe u.a. Wulf 2005, Callaghan/Kernic 2004 oder Moskos/Williams/Segal 2000. angestiegen. Obwohl die Gründe hierfür vielfältig sind, spielte dabei die Verbreitung von Menschenrechts- und Demokratienormen eine wichtige Rolle. Indem sowohl Demokratisierung als auch die Einhaltung der Menschenrechte auf die außenpolitische Agenda westlicher Regierungen gesetzt wurden, konnten letztere die Augen vor massiven Menschenrechtsverletzungen nicht mehr verschließen und sahen sich gezwungen zu handeln. Die Zunahme der verabschiedeten PKOs hatte eine verstärkte Nachfrage nach mehr Truppenkontingenten zur Folge, die oft nicht befriedigt werden konnte bzw. nicht befriedigt werden kann.

Drittens waren es die Vertreter privater Militärfirmen selbst, die die Diskussion um eine Beteiligung ihrer Unternehmen an PKOs in den letzten Jahren angekurbelt hatten. Obwohl der Konflikt im Irak nach wie vor nicht gelöst ist und Tausende von Soldaten und Mitarbeitern der Sicherheitsindustrie beschäftigt, suchen letztere bereits nach neuen Geschäftsfeldern. In den USA haben sich in der International Peace Organisation Association (IPOA) 35 private Militärfirmen zusammengeschlossen. Nicht nur der Name dieses Verbandes, sondern auch der Titel der von ihnen herausgegebenen Zeitschrift (Journal of International Peace Operations, JIPO) zeigt, dass sich diese Unternehmen dem Thema Friedensoperationen zugewandt haben. Beiträge berichten beispielsweise über stattgefundene Kooperationen (»Private Security Companies in Afghanistan. Working Hand-in-Hand with NATO and the Afghanistan Government «, JIPO Vol.3, No. 1, July-August 2007) oder machen Vorschläge, wie aktuelle PKOs von den privaten Sicherheitsagenturen unterstützt werden könnten. So ist Brooks überzeugt, dass PMCs folgende Aufgaben zur Unterstützung der Truppen der Afrikanischen Union übernehmen könnten: (...) providing logistics, base construction, management and operations, medical services, and helicopters and vehicles for (...) troops. (...) The possibilities include unmanned aerial vehicles to provide reconnaissance, enhanced airmobile capabilities to cover more territory, and private security that could protect at-risk villages and camps for the internally displaced « (Brooks 2006: 4). Dies zeigt die Überzeugung der IPOA, wonach PMCs in sehr unterschiedlichen Szenarien und für verschiedenste Zwecke in Friedensmissionen eingesetzt werden könnten. Mit großer Wahrscheinlichkeit ist es aber nicht nur der Geschäftstrieb oder das humanitäre Gewissen dieser Unternehmen, Aufgaben multilateraler Friedensmissionen auszuführen. Die Mitarbeiter von PMCs werden von Kritikern regelmäßig als moderne Söldner oder als »Kriegshunde« bezeichnet - ein Image, das korrekte Auftraggeber abstößt. Wenn die UNO, die NATO oder ein westlicher demokratischer Staat als Referenz angeführt werden kann, ist das der Reputation - und damit einer Gewinnsteigerung - stark förderlich.

Zusammenfassend lässt sich konstatieren, dass die drei angeführten Triebkräfte, die die Diskussionen um eine mögliche Beteiligung privater Militärfirmen an multilateralen PKOs begründeten, ineinander greifen. Mit dem Zusammenbruch der bipolaren Welt wurden die staatlichen Armeen verkleinert und infolgedessen militärisches Personal freigestellt. Die militärischen Aufgaben veränderten sich jedoch stark, indem einerseits neue Aufgaben hinzukamen und andererseits eine starke Technologisierung Einzug hielt. Folglich konnten die 
reduzierten Streitkräfte den neuen Aufgaben nur ungenügend nachkommen und mussten Teile davon an private Unternehmen abgeben. Damit etablierte sich ein neuer Wirtschaftszweig, der auf Grund der stetig wachsenden Zahl an potenziellen Aufgaben umso attraktiver wurde und infolgedessen anwuchs. Indem die PMCs immer professionellere (und umfassendere) Dienstleistungen anbieten, geraten die staatlichen Armeen umso mehr in Versuchung - oder gar in Bedrängnis -, gewisse Tätigkeiten der Sicherheitsindustrie zu überlassen.

\section{Argumente für einen Einsatz von PMCs}

Befürworter eines Engagements von PMCs in Friedensoperationen führen vor allem drei Punkte an, in welchen sie den multilateralen PKOs überlegen sind: Sie sind schneller am Einsatzort, können den Auftrag kostengünstiger und professioneller ausführen.

Ein anerkanntes Problem der UNO-Friedensmissionen besteht darin, dass mehrere Monate zwischen einem Sicherheitsratsbeschluss und der endgültigen Stationierung der Truppenkontingente verstreichen. Obwohl seit 1994 beziehungsweise 1996 zwei Instrumente im Sinne von Schnellen Eingreiftruppen geschaffen wurden (UN Stand-by Arrangements System [UNSAS] und die Multinational Standby High Readiness Brigade for United Nations Operations [SHIRBRIG]), sind beide nach wie vor nicht im ursprünglich vorgesehenen Sinne eingesetzt worden. Im Gegensatz zur SHIRBRIG, welche für bisher sechs Einsätze zwischen drei und 895 Personen zur Verfügung gestellt hat, haben sich in Bezug auf UNSAS erst zwei Staaten für den » Rapid Deployment Level« verpflichtet. PMCs zielen darauf ab, diese Lücke zu schließen, indem sie beteuern in der Lage zu sein, ihre Mitarbeiter innerhalb von zwei bis sechs Wochen im entsprechenden Krisengebiet zu stationieren. In diesem Sinne könnten private Militärfirmen quasi als Ersatz bislang unzureichend einsetzbarer »Rapid Reaction Forces «, unter UNOKommando eingesetzt werden - bis die UNO-Friedensmission zusammengestellt ist und vor Ort eintrifft.

Der Bericht des kanadischen UNO-Blauhelmkommandeurs der UNAMIR, Roméo Dallaire, zeigt verschiedene Defizite in der Ausbildung, der Ausstattung und der Kooperation der verschiedenen Truppenkontingente auf (Dallaire 2003). Dass die Probleme, mit welchen die UNAMIR in Ruanda 1994 konfrontiert war, nicht die Ausnahme sind, ist nicht von der Hand zu weisen. Neben den zum Teil besorgniserregenden Mängeln in der Ausbildung führen nicht nur sprachliche, sondern auch kulturelle Unterschiede zu Verständigungsproblemen, die verheerende Auswirkungen haben können. Aus diesem Grund wirbt die Firma MPRI für ihre Dienstleistungen damit, dass ein Großteil ihrer Mitarbeiter ehemalige US-amerikanische Soldaten sind und sie deshalb Professionalität gewährleisten können.

Schließlich wird die Diskussion auch auf einer monetären Ebene geführt. Doug Brooks, Präsident der IPOA, erklärte in einem Interview mit der Deutschen Welle, dass eine von PMCs geführte PKO schätzungsweise lediglich zehn bis 25 Prozent von dem kosten würde, was die UNO für eine identische
Mission aufbringen müsste. Die Gründe dafür dürften vor allem darin liegen, dass viele Firmen mit freien Mitarbeitern zusammenarbeiten und so keine fixen "Standkosten « haben und ihre Dienstleistungen flexibel dem Auftrag entsprechend zusammenstellen können.

Neben diesen drei Argumenten, die vorwiegend von Vertretern der Industrie vorgebracht werden, ist das Thema des politischen Willens zu bedenken. Dieser ist für jede Friedensmission, die mit einem UNO-Mandat versehen ist, zentral. Wenn sich die ständigen Sicherheitsratsmitglieder nicht auf eine Resolution einigen, dann ist der Weg zu einer PKO versperrt. Ist die erste Hürde der politischen Willensbildung jedoch genommen und es wird eine Friedensmission beschlossen, dann folgen meistens intensive innenpolitische Diskussionen um die Entsendung von Truppen - dies zumindest in denjenigen Staaten, in welchen das Parlament über die Entsendung der Streitkräfte ins Ausland abstimmen muss. Diese Debatten können erstens inhaltlich zu sehr unterschiedlichen Ergebnissen führen, indem nicht nur die Anzahl der zu entsendenden Soldatinnen und Soldaten der politischen Meinung und Machtverhältnisse unterworfen ist, sondern auch das Festhalten an bestimmten Einsatzgebieten (so beharrte Deutschland auf der Stationierung seiner ISAF-Truppen im »sicheren« Norden Afghanistans).

Geht es um eine Verlängerung eines Mandats, das vom Parlament abgesegnet werden muss, dann kann es von zentraler Bedeutung sein, ob eigene Verluste zu verzeichnen waren. In diesem Zusammenhang spielt die öffentliche Meinung eine wichtige Rolle. Ist die Gesundheit und das Leben von den im Einsatz stehenden Truppen bedroht, dann sehen sich Politiker in westlichen Demokratien mit einer Öffentlichkeit konfrontiert, die deswegen möglicherweise einen Abzug der Truppen fordert. Je nachdem wie die innenpolitischen Konstellationen sind, ist vorstellbar, dass sich politische Entscheidungsträger dem Druck der Gesellschaft beugen und das Peacekeeping-Engagement beenden. Die Bilder der getöteten, verstümmelten und aufgehängten Leichen von Blackwater-Mitarbeitern gingen 2004 um die Welt und lösten zwar Entsetzen aus - jedoch waren die Reaktionen darauf keineswegs mit denjenigen aus dem Jahre 1993 zu vergleichen, als die US-Regierung ihren Rückzug aus Somalia bekannt gab, nachdem amerikanische Soldaten getötet und deren Leichen durch Mogadischu geschleift worden waren. Es wäre folglich gerade für diejenigen Staaten attraktiv, auf Angehörige privater Militärfirmen zurückzugreifen, die zwar aus politisch-ethischen Gründen international gegen Menschenrechts- oder Kriegsverbrechen vorgehen wollen, jedoch für das im Rahmen der PKOs geforderte militärische Engagement in ihrer Gesellschaft keine Zustimmung finden.

Zweitens führen derartige parlamentarische Debatten nicht zu schnellen Entscheidungen. Es kann Wochen, wenn nicht Monate dauern, bis feststeht, ob und in welchem Umfang Truppen des jeweiligen Staates einer PKO zur Verfügung gestellt werden. Doug Brooks fasste in einem Artikel, der 2005 im »The National Interest « erschien, das Availability-Kriterium wie folgt zusammen: »UN missions are only as good as the UN members that support those missions with their own citizens. Western nations, to the detriment of UN peacekeeping, have 
largely abrogated this responsibility« (Brooks 2005). Wenn der politische Wille für eine Unterstützung einer PKO mit eigenen Truppen fehlt - und dies aus vorwiegend innenpolitischen Gründen -, stehen keine Peacekeeper zur Verfügung.

\section{Argumente gegen einen Einsatz von PMCs}

Die Kritikpunkte, die gegen potenzielle Engagements privater Militärfirmen in Friedensmissionen sprechen, sind sehr breit gefächert, weshalb hier nur auf die wichtigsten eingegangen wird. Erstens entsteht mit der Vergabe von Aufgaben grundsätzlich eine sogenannte Principal-agent-Situation, bei der generell Schwierigkeiten der Kontrolle der Aufgabenerledigung entstehen. Ist der Beauftragte nun in einem Konfliktgebiet tätig, wo es täglich zu außerordentlichen Situationen kommt, sind die Monitoring- und Kontrollmöglichkeiten noch schwieriger zu gestalten. Es besteht also die Gefahr, dass sich der Agent verselbstständigt und der Auftraggeber weder weiß, was sein Bevollmächtigter macht, noch einen Einfluss auf dessen Aktivitäten ausüben kann. Damit zusammenhängend besteht ein Problem in der vertraglichen Bindung. Die Konsequenzen einer einseitigen Vertragsauflösung oder eines Vertragsbruchs seitens einer PMC sind schwer berechenbar - könnten aber eine destabilisierende Wirkung sowohl auf eine Friedensmission als auch auf einen Friedensprozess haben.

Zweitens ist die strafrechtliche Verantwortung von PMC-Mitarbeitern nach wie vor nicht geklärt. Verschiedene Menschenrechtsvergehen von Angehörigen privater Militärfirmen, die keine strafrechtlichen Konsequenzen nach sich zogen, waren in den letzten Jahren zu verzeichnen. Das Problem hierbei ist einerseits die rechtliche Grauzone in der viele Unternehmen arbeiten. Andererseits gibt es für gewisse Verbrechen durchaus eine individuelle völkerstrafrechtliche Verantwortlichkeit, an deren Durchsetzung es jedoch mangelt. Solange eine stärkere nationale und internationale Regulierung sowie eine konsequentere Anwendung der entsprechenden Normen fehlen, sollte der Einsatz einer PMC äußerst gut überlegt sein.

Drittens besteht die Gefahr, dass ein Engagement einer PMC durch die UNO scheinheilig wirkt. Seit 1989 existiert eine UNO-Konvention gegen die Rekrutierung, den Einsatz, die Finanzierung und das Training von Söldnern. Obwohl in der Literatur darauf hingewiesen wird, dass private Militärfirmen nicht als herkömmliche Söldner eingestuft werden können, werden vor allem zwielichtige Unternehmen mit einem (neuen) Söldnertum verglichen. Dementsprechend kann die Reputation der Vereinten Nationen - oder auch die anderer internationaler Organisationen, die PKOs mit einem UNOMandat durchführen - leiden, wenn sie als Auftraggeberin von »Söldner-Firmen« wahrgenommen wird.

Viertens existiert mit der NATO Response Force (NRF) eine Schnelle Eingreiftruppe, die das Argument langer Anlaufzeiten entkräftet. Die NRF wurde nach einem Vorstoß der USA im November 2002 auf dem NATO-Gipfel in Prag ins Leben gerufen. Ziel der NRF ist es, erste Einheiten innerhalb von fünf Tagen in ein Krisengebiet zu verlegen, um in den nachfolgenden 30 Tagen unabhängig Einsätze durchführen zu können. Das Aufgabenspektrum reicht von Katastrophenhilfe über Evakuierungsoperationen bis hin zu kompletten Militärinterventionen. Die NRF basiert auf einem Rotationsprinzip, bei welchem die Staaten für jeweils sechs Monate eine bestimmte Anzahl von Streitkräften für die NRF verpflichtend abstellen. Im Mai 2007 wurden im Baltikum verschiedene Übungen mit Land-, See- und Luftstreitkräften mit insgesamt 10.000 Soldaten aus 19 Ländern durchgeführt, die zeigten, dass die Strukturen funktionieren. Wenn die NFR auch für große humanitäre Interventionen eingesetzt wird, so ist das Argument, dass die privaten Militärfirmen schneller als multilaterale Truppen verfügbar sind, entkräftet.

\section{Diskussionen in einzelnen Staaten}

Die Diskussionen um den Einsatz von Akteuren aus der Sicherheitsindustrie beschränkt sich nicht auf wissenschaftliche Zirkel, engagierte Menschenrechts-NGOs und PMC-Industrie. Es ist in den letzten Jahren auch in einzelnen Staaten diesbezüglich zu substanziellen Debatten gekommen.

Es erstaunt nicht, dass die Diskussion um private Sicherheitsdienstleister in den USA weiter fortgeschritten sind als in anderen Ländern, zumal das militärische US-Engagement alleine im Irak in hohem Maße auf PMCs zurückgreift. Einerseits gehören die USA in Bezug auf Regulierungen zu den wenigen Staaten, die eine Bewilligungspflicht für Sicherheitsfirmen kennen. Der US Arms Export Control Act wurde bereits 1968 eingeführt und ist in den 1980er Jahren erweitert worden, indem neben Waffenexporten auch der Export militärischer Dienstleistungen reguliert wird. Amerikanische Firmen müssen sich beim State Department registrieren und erhalten nach einer Prüfung eine Lizenz. Rechtliche Rahmenbedingungen bestehen also - es geht jedoch auch hier um die Umsetzung, Durchsetzung und Kontrolle der bestehenden Regulierungen. Andererseits haben bereits vor einigen Jahren die ersten Debatten im Kongress stattgefunden, die den Einsatz von privaten Anbietern - auch bei PKO - zum Inhalt hatten. Im Herbst 2004 sprach sich der republikanische Abgeordnete Ed Royce in einem Kongress-Hearing für den Einsatz von PMCs in UNOPeacekeeping-Operationen aus. Interessanterweise wurde $\mathrm{zu}$ dieser Anhörung auch der Präsident der IPOA, Doug Brooks, eingeladen, was zeigt, dass die Sicherheitsindustrie als Akteur wahrgenommen und als wichtig erachtet wird. Und erst Ende Juni 2007 ist dem US-Kongress ein neuer Bericht zugestellt worden, welcher die Situation der Sicherheitsanbieter im Irak diskutiert. $^{5}$

2002 hat die britische Regierung ihrerseits mit dem sogenannten Greenpaper Aufsehen erregt, das konstatiert: »The question of employing private military companies in wider roles for the $\mathrm{UN}$ is something which needs debate (House of Commons 2002: 19). Das Greenpaper streicht die Probleme der UNO im Rekrutieren von PK-Truppen heraus und verweist unter Bezug auf das Engagement der US-amerikanischen Firmen DynCorp und Pacific A\&E auf dem Balkan, darauf, dass PKOs mit der

\footnotetext{
5 Siehe »CRS Report for Congress. Private Security Contractors in Iraq: Background, Legal Status, and Other Issues « unter: http://www.fas.org/sgp/crs/ natsec/RL32419.pdf.
} 
privaten Option billiger und effektiver würden. Schließlich wird die Meinung vertreten, dass die Probleme, die mit der Beschäftigung von PMCs aufkämen (vor allem die Problematik der accountability), mit einem Auftraggeber wie der UNO oder einer regionalen Organisation nicht auftreten würden - wieso dem so sein würde, wird nicht weiter ausgeführt.

In Deutschland hat 2005 die FDP-Fraktion zusammen mit weiteren Abgeordneten, im Bundestag eine Große Anfrage eingebracht, die Antworten auf potenzielle Auslagerungen von Aufgaben des Sicherheits- und Militärbereichs verlangt. Im Bericht der Bundesregierung hieß es, dass grundsätzlich hoheitliche Sicherheitsaufgaben nicht an Private abgegeben werden können. Zu sogenannten Beleihungen kann es nur in äußerst speziellen und einzigartigen Fällen kommen, wozu es zudem eines Gesetzes bedarf. Auf die Frage, ob »militärische« Aufgaben an Private abgegeben werden können, gibt die Bundesregierung einerseits eine klare Antwort: »)Militärische< Tätigkeiten im In- oder Ausland können (...) nicht auf private Unternehmen übertragen werden « (Deutscher Bundestag 2005: 5). Andererseits wird eingeräumt, dass »militärische« Aufgaben nicht definiert sind - die Bundesregierung umreißt diese aber als Aufgaben, das dem Gewaltmonopol zugeordnet werden, welches wiederum »(...) Einsätze [beinhaltetet], die hoheitlich-exekutivische Eingriffe mit Anordnungs- und Zwangsbefugnissen (...) (ibid.) zur Folge haben. Indem Auslandseinsätze der Bundeswehr ebenfalls zu den Aufgaben gezählt werden, die hoheitlich-exekutive Eingriffe ausführen, würde dies bedeuten, dass Deutschland einer Kooperation Bundeswehr/PMCs - auch in Friedensmissionen - nicht zustimmen würde.

Auch die schweizerische Regierung hat sich dem Thema der privatisierten Sicherheit angenommen und 2005 einen »Bericht zu den privaten Sicherheits- und Militärfirmen « verfasst, der auf Grund parlamentarischer Vorstöße ausgearbeitet wurde. Eine mögliche Zusammenarbeit zwischen PMCs und der schweizerischen Armee ist kein spezielles Thema - schon gar nicht im Ausland. Es wird dabei lediglich eine generelle Absicht festgehalten, dass Privatisierungen von Sicherheits- und Polizeitätigkeiten, wenn überhaupt, nur in Randbereichen möglich sind. Was die Dienstleistungen von schweizerischen Unternehmen im Ausland betrifft, ist der Bundesratsbericht nicht klar. Er anerkennt aber - und die Exekutive sei auch bereit zu prüfen -, dass es für diejenigen Firmen eine bundesrechtliche Bewilligungs- und Registrierungspflicht geben sollte, die von der Schweiz aus Aufgaben in Krisen- und Konfliktgebieten ausüben. Obwohl eine Untersuchung innerhalb der Bundesverwaltung zeigte, dass die Delegation von Aufgaben an Sicherheitsanbieter eine »eher untergeordnete Rolle« (Bundesrat 2005: 2) spiele, sehe sich die Schweiz aus verschiedenen Gründen dazu verpflichtet, sich international für einen Dialog einzusetzen und internationale Regulierungsansätze zu erarbeiten. ${ }^{6}$

6 Siehe »Swiss initiative on private military and security companies«, unter: www.eda.admin.ch/psc.

\section{Schlussbemerkungen}

Die Debatte über den Einsatz von PMCs in multilateralen Friedensmissionen wird äußerst kontrovers geführt. Die Befürworter eines Engagements von PMCs führen an, dass die privaten Anbieter kostengünstiger und professioneller arbeiten und dass sie schneller einsetzbar seien. Gegen das erste Argument - die oft erwähnte angebliche Kostenersparnis - ist einzuwenden, dass das Sparpotenzial häufig überschätzt wird, da vielfach die unterbreiteten Angebote und nicht die tatsächliche Umsetzung der Aufgaben mit den Kosten einer PKO verglichen werden. Sogar der amerikanische Rechnungshof stellte Ende der 1990er Jahre fest, dass es nicht möglich ist, zu überprüfen, ob es bei getätigten Auslagerungen überhaupt zu Einsparungen gekommen sei (Wulf 2005: 190-191). Zweitens kann einer Friedensmission nicht per se Professionalität und Qualität abgesprochen werden. Werden materielle und personelle Ressourcen nur unzureichend von den politischen Entscheidungsträgern bewilligt, ist die erfolgreiche Durchführung einer PKO gefährdet - das gleiche würde aber auch für eine Operation privater Sicherheitsdienstleister gelten. Drittens ist zwar nicht zu bestreiten, dass Mitarbeiter von PMCs schneller an einen Einsatzort verlegt werden können als UNO-Truppen. Dem könnte die internationale Gemeinschaft jedoch entgegenwirken, indem sie die bestehenden Institutionen und Ressourcen stärker einsetzt - sei dies mit einer Stärkung der UNSAS oder einem konkreten Einsatz der NATO Response Force. Als viertes Argument für einen Einsatz von PMCs wird die Verfügbarkeit angeführt. Dass diverse multilaterale Friedensoperationen an akutem Personalmangel leiden, liegt vorwiegend am fehlenden politischen Willen einiger westlicher Staaten, eigene Truppen für Friedensmissionen bereitzustellen. Die mangelnde Einsatzbereitschaft ist vor allem auf innenpolitische Gründe zurückzuführen. Sind verletzte und gefallene eigene Soldaten zu beklagen, fordern womöglich Teile der Öffentlichkeit ein Ende des Friedenseinsatzes. Je nachdem, welche politischen Verhältnisse vorherrschen, erwägen Politiker auf Grund der öffentlichen Meinung einen Abzug der eigenen Soldaten. Steht eine Gesellschaft bereits vor dem eigentlichen Einsatz dem Vorhaben kritisch oder ablehnend gegenüber, dann werden höchstwahrscheinlich keine nationalen Soldaten in einen Auslandseinsatz geschickt. Natürlich ist auch eine Auslagerung von Friedenssicherungsaufgaben an private Anbieter eine Frage des politischen Willens. Es geht bei den Debatten um Auslandseinsätze aber um das Wohl der Angehörigen der nationalen Streitkräfte und nicht um ordnungspolitische und staatsrechtliche Diskussionen, inwiefern die Auslagerung von Sicherheitsaufgaben überhaupt zulässig ist. Am Schicksal fremder Soldaten oder gar privater Sicherheitsanbieter nimmt eine breite Öffentlichkeit nicht in demselben Ausmaß Anteil wie am Los der eigenen Armeeangehörigen. Wenn Staaten PMCs beschäftigen, können sie den internationalen Forderungen nach mehr Engagement nachkommen und gleichzeitig Debatten um den Einsatz der eigenen Truppen vermeiden. In diesem Sinne ist das Availability-Kriterium ein äußerst wirkungsvolles Argument für den Einsatz von PMCs in Friedenssicherungsoperationen.

Ein entscheidendes Argument gegen das Engagement von PMCs generell, und somit auch gegen deren Beteiligung an 
multilateralen Friedensmissionen, sind die oft inexistenten oder unzureichenden Monitoring- und Kontrollmechanismen. Gut funktionierende Aufsichtsinstrumente sind der Dreh- und Angelpunkt in der Diskussion um die Auslagerung von Aufgaben an PMCs. Sind diese Kontrollmöglichkeiten schlecht ausgestaltet, ist das Auftreten von Missständen und Skandalen, wie die Nichtverfolgung von Menschenrechtsverletzungen oder eines ungerechtfertigten Kostenanstiegs, vorprogrammiert. In der Literatur werden verschiedene Kontrollmöglichkeiten aufgezeigt. So schlagen Schreier und Caparini mehrere Verfahren vor, denen Unternehmen zustimmen müssten, sofern sie integer und kooperationswillig sind. Auf nationaler Ebene sind zwei Elemente hervorzuheben. Erstens kann ein Staat mit einem Lizenzierungssystem die wichtigsten Grundsätze und Regeln für den Einsatz von PMCs festlegen. Es ist dabei vorstellbar, dass neben den klar umrissenen Aufgaben auch die Verpflichtung festgehalten wird, dass Bestimmungen des Völkerrechts eingehalten werden müssen und dass das Unternehmen spontane Inspektionen vor Ort zu akzeptieren habe. Zudem sind auch Auflagen bezüglich der Mitarbeiter einer PMC denkbar, zum Beispiel welchen Anforderungen die Beschäftigten bezüglich Ausbildung genügen müssen. Zweitens ist die Mitwirkung der nationalen Parlamente erforderlich. Die Abgeordneten könnten in einer speziell dafür eingerichteten Kommission bereits beim Lizenzierungsverfahren miteinbezogen und später in den Monitoring- und Kontrollprozess eingebunden werden. Die Kontrollen selbst können von verschiedenen Personen oder Organisationen durchgeführt werden, z.B. durch Botschaftspersonal (Militärattachés), durch speziell dafür eingesetzte nationale Inspektoren oder auch durch eine damit beauftragte NGO (Schreier / Caparini 2005: 116-140).

Die privaten Militärfirmen und ihre Dienstleistungen werden vermehrt von politischen Akteuren in Anspruch genommen, was erahnen lässt, dass zukünftig nicht mehr ausschließlich darüber diskutiert wird, ob Aufgaben - auch des Peacekeeping - an private Sicherheitsdienstleister übertragen werden sollen oder nicht. Wahrscheinlicher ist, dass der entsprechende Diskurs künftig auf die Fragen fokussiert sein wird, welche Aufgaben wie, wofür und an wen ausgelagert werden. Befriedigende Monitoring- und Kontrollmöglichkeiten müssen dabei im Zentrum stehen.

\section{Literatur- und Quellenverzeichnis}

Avant, Deborah 2005: The market for force: the consequences of privatizing security, Cambridge University Press, Cambridge.

Brooks, Doug 2006: Focusing on Sudan, in: Journal of International Peace Operations, Vol.2, No.1, July-August 2006.

Brooks, Doug 2005: Privatized Peacekeeping, in: The National Interest, Summer 2005, http://www.findarticles.com/p/ articles/mi_m2751/is_80/ai_n15696643.

Bundesrat 2005: Bericht des Bundesrats zu den privaten Sicherheits- und Militärfirmen, 2.12.2005.

Bures, Oldrich 2005: Private Military Companies: A Second Best Peacekeeping Option?, in: International Peacekeeping, Vol. 12, No. 4, Winter 2005, pp. 533-546.

Callaghan, Jean M. / Kernic, Franz 2004: Armed Forces and International Security. Global Trends and Issues, Lit, Münster.

Dallaire, Roméo 2003: Shake Hands with the Devil, Random House Canada, Ontario.

Deutscher Bundestag 2005: Antwort der Bundesregierung auf die Anfrage der Drucksache 15/4720, Drucksache 15/5824, 24.6.2005.

House of Commons 2002: Private Military Companies: Options for Regulation, 12.2.2002.

Moskos, Charles C./Williams, John Allen/Segal, David R. 2000: The postmodern military, Oxford University Press, New York.

Pacific Architects and Engineers (PAE) 2007: PAE/HSC Haiti, unter: https://www.paecivpol.com/civ_main.asp?pg= missions-haiti.html\&mnu=5.

Pacific Architects and Engineers (PAE) 2007a: PAE/HSC Liberia, unter: https://www.paecivpol.com/civ_main.asp?pg= missions-liberia.html\&mnu=5.

Schreier, Fred/Caparini, Marina 2005: Privatising Security: Law, Practice and Governance of Private Military and Security Companies, DCAF Occasional Paper No. 6, March 2005.

Singer, Peter W. 2003: Corporate warriors: the rise of the privatized military industry, Cornell University Press, Ithaca.

United Nations 2000: Report of the Panel on United Nations Peace Operations, 21. August 2000, A/55/305 - S/2000/809.

Wulf, Herbert 2005: Internationalisierung und Privatisierung von Krieg und Frieden, Nomos, Baden-Baden. 